\title{
Curcumin improves human umbilical cord derived mesenchymal stem cells survival and promotes motor outcome via ERK Signaling after spinal cord injury
}

\section{Wu Wanjiang}

southwest hospital, third military medical university

\section{Chen Xin}

Third Military Medical University Southwest Hospital Department of Pathology

\section{Chen Yaxing}

southwest hospital, Third Military Medical University

Wang Jie

southwest hospital, Third Military Medical University

\section{Zhang Hongyan}

southwest hospital, Third Military Medical University

Ni Fei

School of Nursing, Third Military Medical University

\section{Ling Chengmin}

southwest hospital, Third Military Medical University

\section{Feng Chengjian}

958th hospital of People's Liberation Army

Jichao Yuan ( $\nabla$ yuanjichao@tmmu.edu.cn )

Third Military Medical University Southwest Hospital

\section{Lin Jiangkai}

southwest hospital, Third Military Medical University

\section{Research}

Keywords: Human umbilical cord-derived mesenchymal stem cells, curcumin, spinal cord injury, antiapoptosis, dual-target therapy

Posted Date: July 7th, 2020

DOI: https://doi.org/10.21203/rs.3.rs-38208/v1 
License: (c) (i) This work is licensed under a Creative Commons Attribution 4.0 International License. Read Full License 


\section{Abstract \\ Background}

Human umbilical cord-derived mesenchymal stem cells (hUC-MSCs) transplantation are assumed as a promising strategy in spinal cord injury (SCl). However, the complex pathological microenvironment after $\mathrm{SCl}$ induces the apoptosis of hUC-MSCs, which limits the clinical application for the cell replacement therapy.

\section{Methods}

In this study, in order to investigate whether combined with curcumin could strengthen the therapeutic effects of hUC-MSCs transplantation for SCl, we mediated the apoptosis of hUC-MSCs with TNF-a and transplanted hUC-MSCs into SCI rats, followed by assessed the anti- apoptosis effect and mechanism of curcumin.

\section{Results}

LDH release test and flow cytometry demonstrated that TNF-a led to the hUC-MSCs apoptosis and curcumin increased survival rate of hUC-MSCs with dose-dependent. In addition, we showed that the phosphorylation levels of ERK, JNK and P38 were up-regulated in the hUC-MSCs apoptosis, while curcumin strengthened the phosphorylation of ERK, but not activated the JNK and P38, which was reversed by p42/44 antagonist U0126. Furthermore, we exhibited that the motor function scores and surviving HNA-positive cells were significantly increased after curcumin combined with hUC-MSCs transplantation therapy 8 weeks post SCl, while U0126 markedly attenuated these phenomenons.

\section{Conclusions}

The aforementioned data confirmed that curcumin suppressed the apoptosis of hUC-MSCs through ERK signal pathway and combined curcumin with hUC-MSCs treatment improved motor function after SCl in rats. The current research provides a strong basis for hUC-MSCs replacement therapy in conjunction with curcumin in the treatment and management of SCl in human.

\section{Introduction}

Spinal cord injury (SCl) leads to severe neurological functional deficit, consequently causes enormous financial and emotional burdens for the patients, relatives and society ${ }^{1}$. The complex pathophysiological processes of $\mathrm{SCl}$ including primary and secondary injury lead to few satisfactory treatment procedure in clinic $^{2}$. Recently, increasing evidences demonstrate that mesenchymal stem cells (MSCs) exhibit 
intriguing immunomodulatory and non-teratogenicity properties, possess strong proliferation and multi potent differentiation capacities ${ }^{3}$. MSCs can maintain regenerative capacity after cryopreservation, improve synaptic transmission, and promote neuronal networks ${ }^{4}$. These properties make MSCs transplantation prime candidate for therapeutic strategy for $\mathrm{SCl}$.

Bone marrow and umbilical cord are rich sources of MSCs ${ }^{5}$. Compared with bone marrow-derived MSCs (BMSCs), umbilical cord-derived MSCs (hUC-MSCs) exhibit considerable advantage including the lack of ethical concerns, low oncogenicity, resistance to viral and bacterial contamination, a fast self-renewal characteristic and a higher immunomodulatory capacity ${ }^{6}$. Therefore, hUC-MSCs serve as preferential seed cells when compared with BMSCs. Researches have reported that the cell replacement therapy with hUC-MSCs improves locomotor outcomes after SCl in animal and preclinical studies ${ }^{7-9}$. The mechanism of hUC-MSCs reveal not only the differentiation into neural lineage cells to repair damaged tissues, but also on their capacity to provide a favorable environment for regeneration ${ }^{10}$. However, the pathological microenvironment after SCI mediates the apoptosis of hUC-MSCs, which limits the therapeutic effects for the cell replacement therapy. Consequently, increased the number of survival hUC-MSCs can strengthen the curative effect for the SCl.

Curcumin (cur) is a diketone compound extracted from the rhizome of curcumae, which possess a strong antioxidant ${ }^{11}$, anti-inflammatory ${ }^{12}$, anti-fibrotic ${ }^{13}$ and anti-tumor ${ }^{14}$ properties. Our laboratory confirmed that curcumin can improve the motor function recovery after $\mathrm{SCl}$ through suppressing astrocyte-induced inflammation and fibrosis ${ }^{15,16}$. In addition, curcumin inhibits the apoptosis of rat kidney and cardiomyocytes ${ }^{17,18}$, indicating that curcumin also sustains an anti-apoptosis role. Nevertheless, it remains unknown whether curcumin can modulate the survival of transplanted hUC-MSCs after SCl.

In the present study, we investigate the effect and mechanism of curcumin on the apoptosis of hUCMSCs mediated by inflammation to confirm the hypothesis that exogenous curcumin combined with hUC-MSCs transplantation can improve motor outcomes, on account of the increased the number of survivable hUC-MSCs via anti-apoptosis by curcumin after $\mathrm{SCl}$.

\section{Material And Methods}

\section{Culture and identification of hUC-MSCs}

The hUC-MSCs were provided by Chongqing Guolian Stem Cell Technology Co., Ltd. The cells were resuscitated with the complete media, which consisted of mesenchymal stem cell basal medium (MSCBM) supplemented with 5\% UltraGROTM-Advanced (AventaCell, HPCFDCRL05). Thereafter, the culture medium was replaced once every two days. The cells were harvested with $80 \%$ fusion and identified by flow cytometry.

2. Cell counting kit-8 (CCK8) 
The hUC-MSCs were cultured in 96 well plates according to 2000 cells / well. Different concentrations of curcumin (cur, St. Louis, MO, Lot: C1386) was conducted in triplicate wells by incubation for $24 \mathrm{~h}$. Then the cells were then incubated in $100 \mu \mathrm{l}$ serum-free medium containing $10 \mu \mathrm{l} \mathrm{CCK-8} \mathrm{(Dojindo} \mathrm{Laboratories,}$ Kumamoto, Japan) at $37^{\circ} \mathrm{C}$ for $2 \mathrm{~h}$, followed by optical density (OD) detection at a wavelength of $450 \mathrm{~nm}$ using amicroplate reader.

\section{Lactate dehydrogenase $(\mathrm{LDH})$ release test}

hUC-MSCs were inoculated into 96 well plates at a density of 2000 cells / well. Different concentrations of TNF-a (Peprotech, 300-01A) were used to interfere of hUC-MSCs $(n<5)$ for 24 hours, then follow by the LDH release test kit (Beyotime,C0017) instructions to assess cell apoptosis ${ }^{19}$.

4. Detection of apoptosis in hUC-MSCs by flow cytometry

hUC-MSCs were divided into four groups: control group, TNF-a group, cur group and TNF-a + cur group. According to the results of LDH release test, $5 \mathrm{ng} / \mathrm{ml}$ TNF-a was selected to induce apoptosis of hUCMSCs, and 4uM curcumin was selected for intervention. In ERK blocking groups, U0126 was used ten minutes before intervention.

\section{Western blotting.}

After different interventions of hUC-MSCs \total protein was extracted. The protein was transferred to polyvinylidene difluoride membrane and incubated with p38 (CST, 8690, 1:1000), JNK (CST, 9252, 1:1000), ERK (CST, 4695, 1:1000), p-P38 (CST, 4511, 1:1000), p-JNK (CST, 4668, 1:1000), p-ERK(CST, 4370, 1:1000), p-Bad (CST, 5284T, 1:1000). Images were developed using a gel imaging system.

\section{Animal and groups}

A total of 180 female Sprague-Dawley rats with a body weight of 230 to $250 \mathrm{~g}$ were were used in this study. The animals were randomly divided into six groups: (1) Sham group ( $n=30),(2) \mathrm{SCl}+V e h$ group $(n=30),(3)$ SCl+cur group ( $n=30)$, (4) SCl+hUC-MSCs group ( $n=30),(5) S C l+c u r+h U C-M S C$ group ( $n=30)$, (5) SCl+cur+hUC-MSCs+U0126 group ( $n=30)$. In the sham group, the spinal cord was exposed but not compressed. The $\mathrm{SCl}$ group was given an equal volume of DMSO after the operation. The $\mathrm{SCl}+$ cur group was treated with $100 \mathrm{mg} / \mathrm{kg}$ cur. The mice in SCl+hUC-MSCs group were transplanted with hUC-MSCs at the injured site after SCl. The SCl+cur+hUC-MSCs group was transplanted with hUC-MSCs combined with $100 \mathrm{mg} / \mathrm{kg}$ cur. The SCl+cur+hUC-MSCs+U0126 group was was transplanted with hUC-MSCs combined with $100 \mathrm{mg} / \mathrm{kg}$ cur and $0.5 \mathrm{mg} / \mathrm{Kg}$ U0126. Cur was given via intraperitoneal injection. The first injection was administered at 30 minutes after the operation and then once a day for a total of 14 days. Non-hUCMSCs transplantation groups were stereotaxic injection of MSC basic medium, hUC-MSCs transplantation groups were transplanted with hUC-MSCs to the injured site in the same way. All animal experiments and care conditions were approved by the Third Military Medical University Committee on Ethics in the Care and Use of Laboratory Animals. 
A SCI model was established with reference to previously described methods ${ }^{15}$. The animals were monitored twice per day for infections, general health and mobility throughout the post-injury survival period. Bladders were expressed twice daily until the mice reached spontaneous micturition.

\section{Transplantation of hUC-MSCs}

Curcumin of $4 \mathrm{uM}$ was used to intervene for 24 hours. The cells were suspended in the basic medium of mesenchymal stem cells at the concentration of $1 * 10^{5} / \mu \mathrm{l}$. The stereotactic apparatus was used to inject, each point (depth of $2 \mathrm{~mm}, 1.5 \mathrm{~mm}, 1 \mathrm{~mm}$ ) were injected the cell suspension (or MSCBM) of $0.8 \mu$ into the injured site at the rate of $1 \mu \mathrm{l} / \mathrm{min}$ for 2 minutes, and the needle was withdrawn slowly. Within three days after transplantation, saline containing 40,000 units of penicillin was intramuscular injected once a day to prevent infection.

\section{Behavioral test}

Eight rats were randomly selected from each group. Basso, Beattie, and Bresnahan (BBB) locomotor rating scale and inclined plate scale were performed on the 3rd day and 2nd to 9th week after $\mathrm{SCl}$. The rats were placed on a circular platform with a diameter of 2 meters for 4 minutes. The recovery of rat hind limb was evaluated by the BBB scale. 2 hour later, the rats were placed on a rectangular board covered with rubber pads. The body axis of the rat was parallel to the bottom of the board, starting at an angle of 30 degrees. When the rats could be stable for more than 5 seconds, the inclined plate angle increased by 5 degrees, and the maximum angle at which the rats could maintain stability was recorded as the score. Both the behavioral evaluation was performed by two people who did not participate in the experiment.

\section{Electrophysiological examination}

The motor evoked potentials (MEP) were examined at the 9th week after injury. The stimulation electrode was inserted subcutaneously into the surface of the skull of rats, and the lower part of the stimulation electrode was corresponding to the motor cortex. The recording electrode was inserted into the tibialis anterior muscle of the hindlimb and the reference electrode was inserted into the dorsal subcutaneous layer near the root of the tail of the rat. The single stimulus with $25 \mathrm{~mA}$ intensity was measured twice at an interval of 1 minute. The amplitudes of the double hindlimb electrodes were measured, and the average values of the four measurements were calculated to obtain the final amplitude of the MEP.

\section{Immunofluorescence}

At the 9th week after $\mathrm{SCl}$, tissues from within $0.5 \mathrm{~cm}$ of the injury region were dissociated and immunofluorescence staining with reference to previously described methods ${ }^{20}$. The positive cells were counted using Image J.

12. Statistical analysis 
All data are presented as the mean \pm standard error. SPSS22.0 software was used for statistical analysis. One-way ANOVA with the appropriate LSD post hoc test was used to compare experimental groups. ${ }^{*} \mathrm{P}$ 0.05 was considered statistically significant.

\section{Results}

1. hUC-MSCs culture and identification

Light microscopy revealed that the cells grew adherently to the wall, and approximately $80 \%$ of the cells were fused in 3 days. When the cells reached 100\% fusion, they became typical fish schools (Fig. 1A). The flow cytometry showed that more than $95 \%$ of the cells expressed the mesenchymal stem cells makers such as CD73, CD90 and CD105, but not expressed the hematopoietic stem cells markers such as CD34, CD45 and HLA-DR (Fig. 1B). These results identified that the cultured adherent cells were hUCMSCs and the purity of the cells met the standard of the experiment.

\section{Curcumin inhibited apoptosis of hUC-MSC mediated by TNF- a}

To determine the optimum concentration of cur, we assessed the cytotoxicity in hUC-MSC with CCK-8 (Fig. 2A). The CCK-8 results showed that there were statistic differences when concentration of cur was $\geq 16 \mu \mathrm{mol} / \mathrm{L}$ as compared with the control group, indicating no cytotoxicity when the concentration of cur was $\leq 8 \mu \mathrm{mol} / \mathrm{L}$. Therefore, we selected the concentration of cur with $1 \mu \mathrm{mol} / \mathrm{L}, 2 \mu \mathrm{mol} / \mathrm{L}, 4 \mu \mathrm{mol} / \mathrm{L}$ in the subsequent experiments.

Further, we assessed the effect of cur on the apoptosis mediated with TNF- a (5 ng/mL) in hUC-MSC. The $\mathrm{LDH}$ release test demonstrated that TNF- $a$ at the concentration of $5 \mathrm{ng} / \mathrm{mL}$ significantly accelerated LDH released by hUC-MSC, whereas cur reversed this phenomenon with dose-dependent (Fig. 2B). Decreased numbers of apoptotic hUC-MSCs were detected by flow cytometry with the increased cur concentration, these data confirmed that cur could reduce the apoptosis of hUC-MSC mediated by TNF- a (Fig. 2C, D).

\section{Curcumin activated ERK/MAPK expression in hUC-MSC, but not the p38/MAPK or JNK/MAPK}

We subsequently examined the expression of MAPK signal pathway in hUC-MSC to evaluate the mechanisms responsible for anti-apoptosis with cur (Fig. 3). WB showed that there was no significant difference on the expression of JNK and P38 in the TNF- a group or the cur group compared to the control group. However, the expression of p-p42/44 was increased in hUC-MSC apoptosis mediated by TNF-a, and cur intervention further enhanced p-p42/44 expression. Furthermore, the expression of $\mathrm{p}$-Bad was also up regulated with cur, which was the downstream protein of ERK signal pathway. These data suggested that ERK signal pathway was involved in the anti-apoptosis effect of cur. Accordingly, we further intervened the different groups of hUC-MSC with the p42/44 antagonist U0126 (Fig. 4). The expression of p-p42/44 were significantly blocked in U0126 intervention groups compared with other groups, followed by reversed the phosphorylation of Bad expression, which was activated by cur. These 
data revealed that cur activated ERK/MAPK expression in the apoptosis of hUC-MSC mediated by TNF-a, but not the p38/MAPK or JNK/MAPK.

4. Curcumin suppressed hUC-MSC apoptosis through ERK/MAPK signal pathway.

Based on the above results, we further detected the anti-apoptosis effect of cur with U0126 intervention (Fig. 5). The quantification by flow cytometry showed that the number of apoptotic hUC-MSC in TNFa+cur group was less than the TNF-a group, indicating cur reduced hUC-MSC apoptosis mediated by TNFa. After U0126 intervention, the number of apoptotic hUC-MSC was increased, suggested blocked the ERK/MAPK signal pathway reversed the anti-apoptosis effect of cur. Consequently, these results substantiated that cur suppressed hUC-MSC apoptosis through ERK/MAPK signal pathway.

5. Curcumin reduced the transplanted hUC-MSC apoptosis in the injured region through ERK/MAPK signal pathway after $\mathrm{SCl}$

To assessed the anti-apoptosis effect of cur\we examined the survival rate of the transplanted hUC-MSC in the injured region 3 days and 8 weeks post transplantation, respectively (Fig. 6). Immunofluorescence staining showed that 3 days post transplantation, a larger number of transplanted hUC-MSC emerged apoptosis in the SCl+ hUC-MSC group. There were more HNA-positive cells and less tunel-positive cells in the SCl+ hUC-MSC+cur group compared with the SCI+ hUC-MSC group, whereas U0126 reversed this phenomenon. After transplantation 8 weeks, there were still part of surviving HNA-positive cells in the $\mathrm{SCl}+\mathrm{hUC}-\mathrm{MSC}$ group, and these cells concentrated in the transplanted position. With cur intraperitoneal injection, the number of HNA-positive cells was increased and the cells distributed surround the injured region. In addition, U0126 intervention suppressed survival rate of the transplanted hUC-MSC. These data confirmed that cur reduced the transplanted hUC-MSC apoptosis in the injured region through ERK/MAPK signal pathway after SCl.

6. Curcumin combined with hUC-MSC significantly promoted the recovery of hindlimb function in rats after SCl.

Finally, in vivo therapeutic effects of cur combined with hUC-MSC transplantation were examined in SCI rats. The BBB scores were 21 points before modeling and 0 point at the third day after $\mathrm{SCl}$, indicating the success of $\mathrm{SCl}$ modeling. The rats underwent transplantation one week after operation. The BBB score (Fig. 7A) and oblique plate score (Fig. 7B) were gradually elevated after SCl in cur group and hUC-MSC transplantation group, in spite of no significant difference between the two groups. There were significant statistical differences among SCl+hUC-MSC, SCl+hUC-MSC+cur and SCl+hUC-MSC+cur+U0126 group. Electrophysiological examination showed that the amplitude of MEP decreased after SCI (Fig. 7C, D). With cur treatment or hUC-MSC transplantation, the amplitude of MEP increased significantly, and the SCl+hUC-MSC+cur group displayed the most prominent amplitude of MEP. Moreover, blocked ERK/MAPK signal pathway with U0126 abolished the therapeutic effect of curcumin combined with hUC-MSC transplantation. These results substantiated that combined with curcumin intraperitoneal injection and 
hUC-MSC transplantation effectively promote the recovery of hind limb function through ERK/MAPK signal pathway, whereas U0126 intervention aggravated the motor function injury.

\section{Discussion}

In current study, we mediated the apoptosis of hUC-MSCs with $5 \mathrm{ng} / \mathrm{ml}$ TNF-a and investigated the effect and mechanism of curcumin on the hUC-MSCs apoptosis. We demonstrated that TNF-a led to the hUCMSCs apoptosis and curcumin increased survival rate of hUC-MSCs with dose-dependent in vitro. In addition, we found that the phosphorylation levels of ERK, JNK and p38 were up-regulated in the hUCMSCs apoptosis, while curcumin strengthened the phosphorylation of ERK, but not activated the JNK and P38, which was reversed by p42/44 antagonist U0126. Furthermore, we verified that the motor function scores and the amplitude of MEP were significantly increased after curcumin combined with hUC-MSCs transplantation therapy in $\mathrm{SCl}$ rats, whereas the recovery on behavioral performance and amplitude of MEP were markedly attenuated by U0126. Accordingly, the data confirmed that curcumin suppressed the apoptosis of hUC-MSCs through ERK signal pathway and combined curcumin with hUC-MSCs treatment improved motor outcome after SCl.

MSCs is a promising therapeutic strategy for $\mathrm{SCl}$ on account of the capacity to differentiate into cells of neural lineage, including neuron, oligodendrocyte, and so forth, to repair damaged tissues ${ }^{21}$. Recently, increasing researches suggested that MSCs served therapeutic effects not only through direct differentiation into replacement nerve cells but primary through immunomodulatory and paracrine mechanisms in the injury region after $\mathrm{SCl}^{22,23}$. Compared to MSCs with other sources, hUC-MSCs were documented faster proliferation, stronger anti-inflammation and a higher immunomodulatory capacity 24,25 . With SCl rats or mouse, hUC-MSCs showed a promising profile of neurotrophic, anti-inflammatory, and anti-apoptotic effects ${ }^{26-29}$. In addition, neuron-like and schwann-Like characteristics were identified after hUC-MSCs transplantation in SCl animal models ${ }^{30,31}$. Despite the aforementioned results in preclinical studies, only few clinical trials demonstrated the minor improvements in some SCI patients ${ }^{32-34}$. Due to the pathology of the microenvironment after $\mathrm{SCl}$, most of the transplanted cells failed to survive, and only a very small fraction of the cells may eventually function to exert the therapeutic purpose ${ }^{24}$. The major limitations in the application of hUC-MSCs in SCl is their low survival rates after graft. Consequently, inhibition the apoptosis of UC-MSCs is a definite method to improve the efficiency of hUCMSCs transplantation.

Curcumin, which is the active component of turmeric, promotes sensorimotor function recovery via antiinflammation ${ }^{35}$, antioxidant ${ }^{36}$, and anti-apoptosis ${ }^{37}$ effects in $\mathrm{SCl}$. There were evidences showed that curcumin improved functional recovery from SCl by combined with the neural stem cells ${ }^{38,39}$ or MSCs treatment ${ }^{40}$. The enhanced therapeutic effect was achieved by modulated the proliferation and differentiation of stem cells and alleviated the inflammation microenvironment in the injury region ${ }^{41}$. However, there was no data displayed the effect of combined curcumin with hUC-MSC in SCI. In the current research, we found that there was no significant difference in hind limb motor score and 
amplitude of MEP between curcumin treatment alone group and hUC-MSC transplantation alone group, whereas the $\mathrm{SCl}+$ hUC-MSC + cur group displayed the most prominent motor score and amplitude of MEP. These data suggested that curcumin in conjunction with hUC-MSC therapy improved the recovery of SCI and enhanced the therapeutic effect of curcumin treatment alone or hUC-MSC transplantation alone. Additionally, we investigated the effect of curcumin on the apoptosis of transplanted UC-MSCs. The LDH release test and flow cytometry showed that TNF-a mediated the transplanted hUC-MSC apoptosis and cur reversed this phenomenon with dose-dependent in vitro. The apoptosis experiment further exhibited curcumin elevated the survival rate of hUC-MSCs in vivo. These data indicated that curcumin reduced the apoptosis of transplanted hUC-MSC. Consequently, the aforementioned results confirmed our hypothesis that increased the survival rate of hUC-MSCs enhanced the therapeutic effects of cells transplantation and improved the hindlimb motor recovery in SCl.

As a potential treatment for $\mathrm{SCl}$, stem cells transplantation has been studied extensively. Increased researches realize that single cell transplantation is difficult to effectively promote the recovery of SCI. In order to overcome the limitations of direct hUC-MSCs transplantation, several strategies have been employed that include pre-transplantation neural differentiation, neurotrophic gene transduction, glial cell co-transplantation, and tissue engineering. However, the glial scar, cystic cavity and the inhibitory cellular environment were still the major limitations in the therapeutic in vivo application of hUC-MSCs ${ }^{42}$. We previous studies had confirmed that the curcumin improved neural outcome after $\mathrm{SCl}$ and combined inhibition the formation of glial scar and the deposition of extracellular matrix through anti-inflammation and anti-fibrosis ${ }^{15,16}$. In the current research, we further testified that curcumin enhanced the survival rate of hUC-MSCs by anti- apoptosis. On the whole, we demonstrated that curucmin break though the limitations of hUC-MSCs application by anti-inflammation, anti-fibrosis and anti- apoptosis effects.

In addition, we further discussed the mechanism of anti-apoptosis of curcumin on hUC-MSCs transplantation in SCl. Extracellular signal regulated kinase cascade is an important signaling pathway in the nervous system, which is the basis of synaptic plasticity, cellular excitability, learning and arousal ${ }^{43}$. The activation of ERK signaling pathway is considered to play a major role in protecting cell survival from apoptosis ${ }^{44}$. Previous studies had exhibited that the activation of ERK pathway promoted Bad phosphorylation and inhibited apoptosis ${ }^{45}$. The results of WB showed that the phosphorylation levels of ERK, JNK and P38 were increased in the hUC-MSCs apoptosis mediated by TNF- $a$, suggested that MAPK signal pathway were involved in apoptosis. Curcumin intervention strengthened the phosphorylation of ERK, but not activated the JNK and P38. What is more, the reduced number of the apoptotic hUC-MSCs by curcumin was reversed by p42/44 antagonist U0126 in vitro. We further verified that the motor function scores and the amplitude of MEP were significantly increased after curcumin combined with hUC-MSCs transplantation therapy in $\mathrm{SCl}$ rats, whereas the recovery on behavioral performance and amplitude of MEP were markedly attenuated by U0126. These data indicated that the mechanism of anti-apoptosis of curcumin on hUC-MSCs transplantation was attained through ERK signal pathway.

\section{Conclusion}


In summary, this study illustrated that curcumin suppressed the apoptosis of hUC-MSCs through ERK signal pathway and combined curcumin with hUC-MSCs treatment improved motor function after SCl in rats. The current research provides a strong basis for hUC-MSCs replacement therapy in conjunction with curcumin in the treatment and management of $\mathrm{SCl}$ in human.

\section{Abbreviations}

BBB: Basso, Beattie, and Bresnahan, BMSCs:bone marrow-derived MSCs, CCK8:cell counting kit-8, cur:curcumin, hUC-MSCs:human umbilical cord-derived mesenchymal stem cells, LDH:lactate dehydrogenase, MAPK, mitogen-activated protein kinase, MEP:motor evoked potentials, MSCBM:mesenchymal stem cell basal medium, MSCs:mesenchymal stem cells, SCl:spinal cord injury

\section{Declarations}

\section{Ethics approval and consent to participate}

All animal experiments and care conditions were approved by the Third Military Medical University Committee on Ethics in the Care and Use of Laboratory Animals

\section{Conflict of Interest}

The authors declare that they have no conflict of interests.

\section{Funding}

This work was supported by grants from the National Natural Science Foundation of China (81571214), Third Military Medical University "Miaopu” talents scheme (XZ-2019-505-064), and Southwest Hospital Science Foundation (SWH2018QNKJ-02).

\section{Authors' contributions}

Conceived and designed the experiments囚LJK, YJC, WWJ. Performed the experiments: WWJ, CX, CYX, ZHY, WJ, NF, LCM. Analyzed the date: YJC, FCJ, LJK. Wrote the paper: WWJ, YJC, LJK.

\section{Availability of data and materials}

The datasets used and/or analyzed during the current study are available from the corresponding author on reasonable request.

\section{References}

1. Global regional. national burden of neurological disorders. 1990-2016: a systematic analysis for the Global Burden of Disease Study 2016. Lancet Neurol 18, 459-80, doi:10.1016/s14744422(18)30499-x (2019). 
2. Tran AP, Warren PM, Silver J. The Biology of Regeneration Failure and Success After Spinal Cord Injury. Physiological reviews. 2018;98:881-917. doi:10.1152/physrev.00017.2017.

3. Stower H. Cell therapy for spinal cord injury. Nat Med. 2018;24:1088. doi:10.1038/s41591-018-0155y.

4. Carr MJ, et al. Mesenchymal Precursor Cells in Adult Nerves Contribute to Mammalian Tissue Repair and Regeneration. Cell Stem Cell. 2019;24:240-56.e249. doi:10.1016/j.stem.2018.10.024.

5. Bartolucci J, et al. Safety and Efficacy of the Intravenous Infusion of Umbilical Cord Mesenchymal Stem Cells in Patients With Heart Failure: A Phase 1/2 Randomized Controlled Trial (RIMECARD Trial [Randomized Clinical Trial of Intravenous Infusion Umbilical Cord Mesenchymal Stem Cells on Cardiopathy]). Circ Res. 2017;121:1192-204. doi:10.1161/circresaha.117.310712.

6. Titomanlio L, et al. Stem cell therapy for neonatal brain injury: perspectives and challenges. Ann Neurol. 2011;70:698-712. doi:10.1002/ana.22518.

7. Yousefifard $M$, et al. Human bone marrow-derived and umbilical cord-derived mesenchymal stem cells for alleviating neuropathic pain in a spinal cord injury model. Stem Cell Res Ther. 2016;7:36. doi:10.1186/s13287-016-0295-2.

8. Ma YH, et al. Perineurium-like sheath derived from long-term surviving mesenchymal stem cells confers nerve protection to the injured spinal cord. Biomaterials. 2018;160:37-55. doi:10.1016/j.biomaterials.2018.01.015.

9. Li X, et al. Transplantation of hUC-MSCs seeded collagen scaffolds reduces scar formation and promotes functional recovery in canines with chronic spinal cord injury. Scientific reports. 2017;7:43559. doi:10.1038/srep43559.

10. Zhu SF, et al. Comparison of cell proliferation, apoptosis, cellular morphology and ultrastructure between human umbilical cord and placenta-derived mesenchymal stem cells. Neurosci Lett. 2013;541:77-82. doi:10.1016/j.neulet.2013.03.018.

11. Júnior MM, et al. Substitution of synthetic antioxidant by curcumin microcrystals in mortadella formulations. Food Chem. 2019;300:125231. doi:10.1016/j.foodchem.2019.125231.

12. Rahimi K, et al. Targeting the balance of $\mathrm{T}$ helper cell responses by curcumin in inflammatory and autoimmune states. Autoimmun rev. 2019;18:738-48. doi:10.1016/j.autrev.2019.05.012.

13. Jin H, et al. Activation of PPARy/P53 signaling is required for curcumin to induce hepatic stellate cell senescence. Cell death disease. 2016;7:e2189. doi:10.1038/cddis.2016.92.

14. Cruz-Correa M, et al. Efficacy and Safety of Curcumin in Treatment of Intestinal Adenomas in Patients With Familial Adenomatous Polyposis. Gastroenterology. 2018;155:668-73. doi:10.1053/j.gastro.2018.05.031.

15. Yuan J, et al. Curcumin inhibits glial scar formation by suppressing astrocyte-induced inflammation and fibrosis in vitro and in vivo. Brain research. 2017;1655:90-103. doi:10.1016/j.brainres.2016.11.002.

16. Yuan J, et al. Curcumin improves neural function after spinal cord injury by the joint inhibition of the intracellular and extracellular components of glial scar. J Surg Res. 2015;195:235-45. 
doi:10.1016/j.jss.2014.12.055.

17. Guerrero-Hue $M$, et al. Curcumin reduces renal damage associated with rhabdomyolysis by decreasing ferroptosis-mediated cell death. FASEB journal: official publication of the Federation of American Societies for Experimental Biology. 2019;33:8961-75. doi:10.1096/fj.201900077R.

18. Yao Q, et al. Curcumin protects against diabetic cardiomyopathy by promoting autophagy and alleviating apoptosis. J Mol Cell Cardiol. 2018;124:26-34. doi:10.1016/j.yjmcc.2018.10.004.

19. Yuan J, et al. M2 microglia promotes neurogenesis and oligodendrogenesis from neural stem/progenitor cells via the PPARgamma signaling pathway. Oncotarget. 2017;8:19855-65. doi:10.18632/oncotarget.15774.

20. Ran H, et al. Adenosine A2A Receptors in Bone Marrow-Derived Cells Attenuate Cognitive Impairment in Mice After Chronic Hypoperfusion White Matter Injury. Translational stroke research. 2020. doi:10.1007/s12975-019-00778-9.

21. Caplan Al. Why are MSCs therapeutic? New data: new insight. J Pathol. 2009;217:318-24. doi:10.1002/path.2469.

22. Dasari VR, Veeravalli KK, Dinh DH. Mesenchymal stem cells in the treatment of spinal cord injuries: A review. World journal of stem cells. 2014;6:120-33. doi:10.4252/wjsc.v6.i2.120.

23. Vismara I, Papa S, Rossi F, Forloni G, Veglianese P. Current Options for Cell Therapy in Spinal Cord Injury. Trends Mol Med. 2017;23:831-49. doi:10.1016/j.molmed.2017.07.005.

24. Moya A, et al. Human Mesenchymal Stem Cell Failure to Adapt to Glucose Shortage and Rapidly Use Intracellular Energy Reserves Through Glycolysis Explains Poor Cell Survival After Implantation. Stem Cells. 2018;36:363-76. doi:10.1002/stem.2763.

25. Jeon YJ, Kim J, Cho JH, Chung HM, Chae Jl. Comparative Analysis of Human Mesenchymal Stem Cells Derived From Bone Marrow, Placenta, and Adipose Tissue as Sources of Cell Therapy. Journal of cellular biochemistry. 2016;117:1112-25. doi:10.1002/jcb.25395.

26. Caron I, et al. A new three dimensional biomimetic hydrogel to deliver factors secreted by human mesenchymal stem cells in spinal cord injury. Biomaterials. 2016;75:135-47. doi:10.1016/j.biomaterials.2015.10.024.

27. Dasari VR, et al. Neuronal apoptosis is inhibited by cord blood stem cells after spinal cord injury. J Neurotrauma. 2009;26:2057-69. doi:10.1089/neu.2008-072510.1089/neu.2008.0725.

28. Wang $\mathrm{N}$, et al. Collagen scaffold combined with human umbilical cord-derived mesenchymal stem cells promote functional recovery after scar resection in rats with chronic spinal cord injury. $\mathrm{J}$ Tissue Eng Regen Med. 2018;12:e1154-63. doi:10.1002/term.2450.

29. Nakajima $\mathrm{H}$, et al. Transplantation of mesenchymal stem cells promotes an alternative pathway of macrophage activation and functional recovery after spinal cord injury. J Neurotrauma. 2012;29:1614-25. doi:10.1089/neu.2011.2109.

30. Yan-Wu G, et al. Human umbilical cord-derived Schwann-like cell transplantation combined with neurotrophin-3 administration in dyskinesia of rats with spinal cord injury. Neurochem Res. 2011;36:783-92. doi:10.1007/s11064-011-0402-9. 
31. Kuh SU, Cho YE, Yoon DH, Kim KN, Ha Y. Functional recovery after human umbilical cord blood cells transplantation with brain-derived neutrophic factor into the spinal cord injured rat. Acta Neurochir (Wien). 2005;147:985-92. doi:10.1007/s00701-005-0538-y. discussion 992.

32. Zhu H, et al. Phase I-II Clinical Trial Assessing Safety and Efficacy of Umbilical Cord Blood Mononuclear Cell Transplant Therapy of Chronic Complete Spinal Cord Injury. Cell Transplant. 2016;25:1925-43. doi:10.3727/096368916×691411.

33. Yao L, et al. Human umbilical cord blood stem cell transplantation for the treatment of chronic spinal cord injury: Electrophysiological changes and long-term efficacy. Neural regeneration research. 2013;8:397-403. doi:10.3969/j.issn.1673-5374.2013.05.002.

34. Rohde E, Pachler K, Gimona M. Manufacturing and characterization of extracellular vesicles from umbilical cord-derived mesenchymal stromal cells for clinical testing. Cytotherapy. 2019;21:581-92.

35. Krupa P, et al. Nano-formulated curcumin (Lipodisq ${ }^{T M}$ ) modulates the local inflammatory response, reduces glial scar and preserves the white matter after spinal cord injury in rats. Neuropharmacology. 2019;155:54-64. doi:10.1016/j.neuropharm.2019.05.018.

36. Lee YS, et al. Effect of curcumin on the inflammatory reaction and functional recovery after spinal cord injury in a hyperglycemic rat model. The spine journal: official journal of the North American Spine Society. 2019. doi:10.1016/j.spinee.2019.07.013.

37. Li W, Yao S, Li H, Meng Z, Sun X. Curcumin promotes functional recovery and inhibits neuronal apoptosis after spinal cord injury through the modulation of autophagy. J Spinal Cord Med, 1-9, doi:10.1080/10790268.2019.1616147 (2019).

38. Bang WS, et al. Curcumin Increase the Expression of Neural Stem/Progenitor Cells and Improves Functional Recovery after Spinal Cord Injury. J Korean Neurosurg Soc. 2018;61:10-8. doi:10.3340/jkns.2017.0203.003.

39. Ormond DR, et al. Stem cell therapy and curcumin synergistically enhance recovery from spinal cord injury. PloS one. 2014;9:e88916. doi:10.1371/journal.pone.0088916.

40. Ruzicka J, et al. Anti-inflammatory compound curcumin and mesenchymal stem cells in the treatment of spinal cord injury in rats. Acta Neurobiol Exp. 2018;78:358-74.

41. Requejo-Aguilar R, et al. Combined polymer-curcumin conjugate and ependymal progenitor/stem cell treatment enhances spinal cord injury functional recovery. Biomaterials. 2017;113:18-30. doi:10.1016/j.biomaterials.2016.10.032.

42. Chhabra HS, Sarda K. Stem cell therapy in spinal trauma: Does it have scientific validity? Indian journal of orthopaedics 49, 56-71, doi:10.4103/0019-5413.143913.

43. Szczurkowska J, et al. NEGR1 and FGFR2 cooperatively regulate cortical development and core behaviours related to autism disorders in mice. Brain. 2018;141:2772-94. doi:10.1093/brain/awy190.

44. Yan W, et al. C1q/Tumor Necrosis Factor-Related Protein-9 Regulates the Fate of Implanted Mesenchymal Stem Cells and Mobilizes Their Protective Effects Against Ischemic Heart Injury via 
Multiple Novel Signaling Pathways. Circulation. 2017;136:2162-77.

doi:10.1161/circulationaha.117.029557.

45. Dahl EF, Wu SC, Healy CL, Perry J, O'Connell TD. ERK mediated survival signaling is dependent on the Gq-G-protein coupled receptor type and subcellular localization in adult cardiac myocytes. J Mol Cell Cardiol. 2019;127:67-73. doi:10.1016/j.yjmcc.2018.11.020.

Figures

A

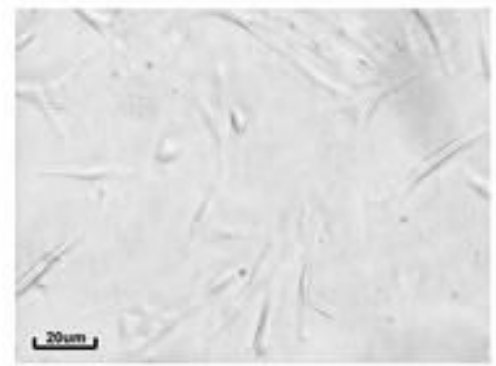

$1 d$

B
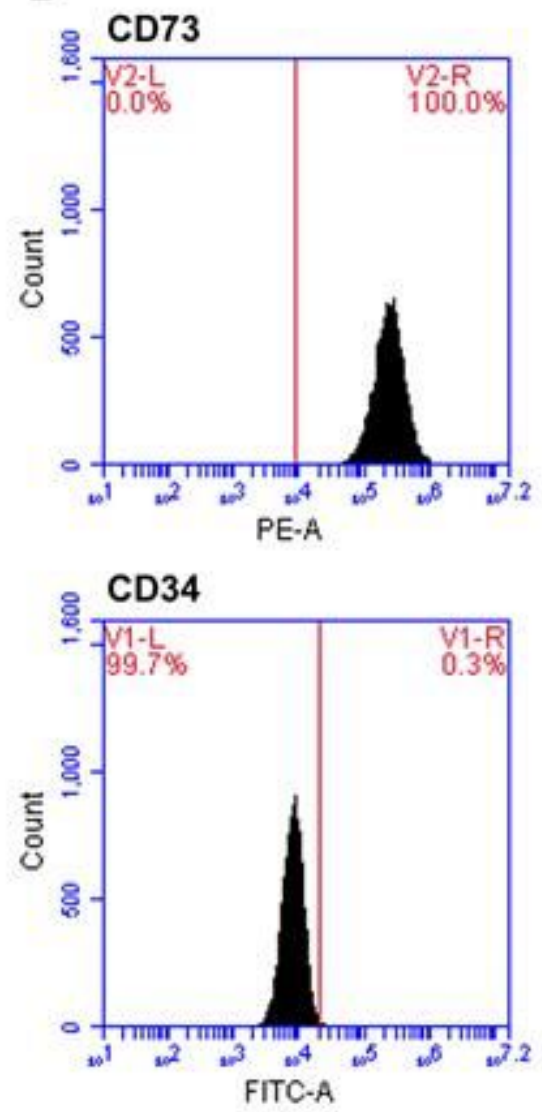

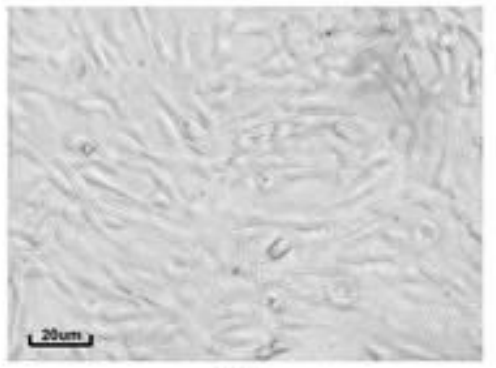

$3 d$
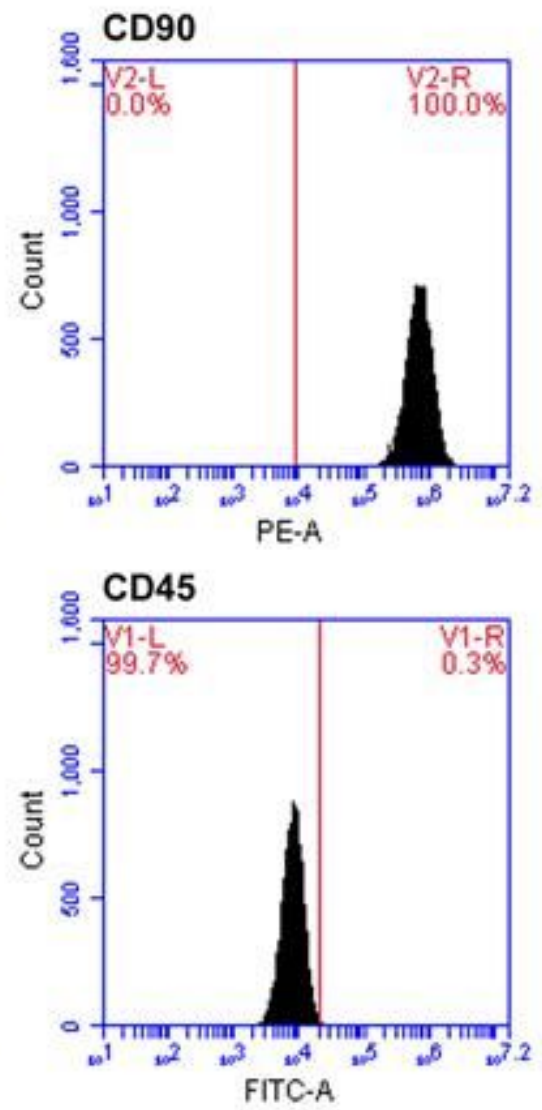

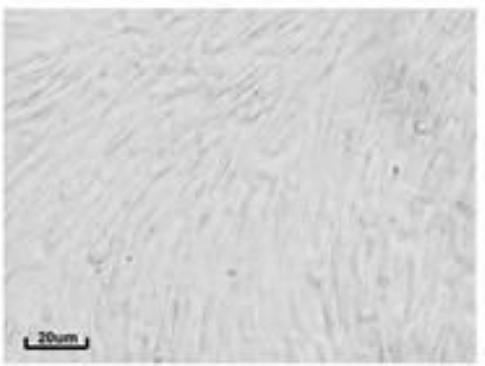

6d
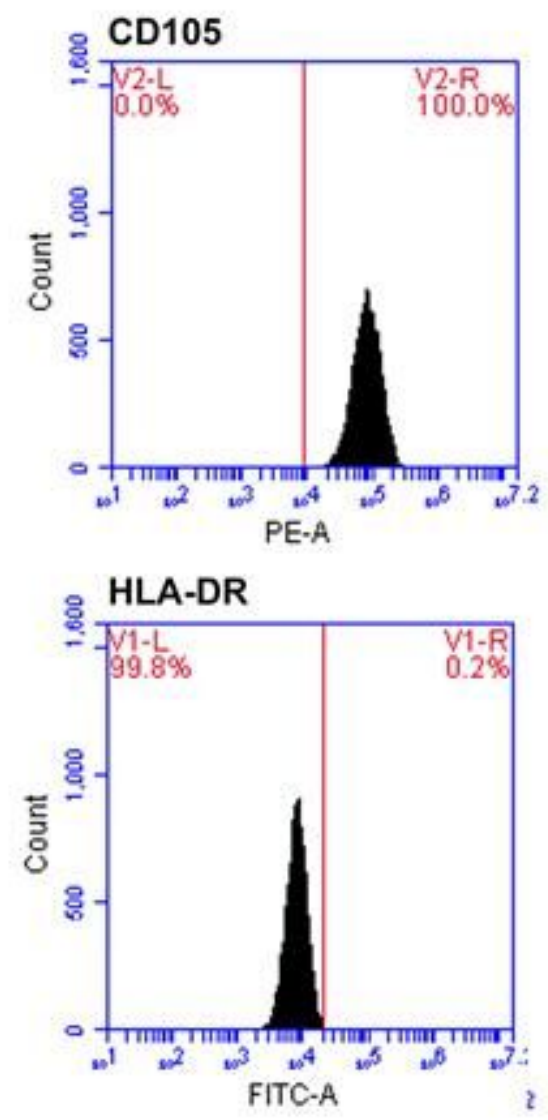

\section{Figure 1}


hUC-MSCs identification. A. Light microscopy showed the representative morphology of hUC-MSCs on day 1 , day 3 and day 6 post cultured in vitro. B. The flow cytometry revealed that the phenotypes of mesenchymal stem cells were positive for CD73, CD90 and CD105, while the phenotypes of hematopoietic stem cells were negative for CD34, CD45 and HLA-DR. bar=20 $\mu \mathrm{m}, \mathrm{N}=5 /$ group.
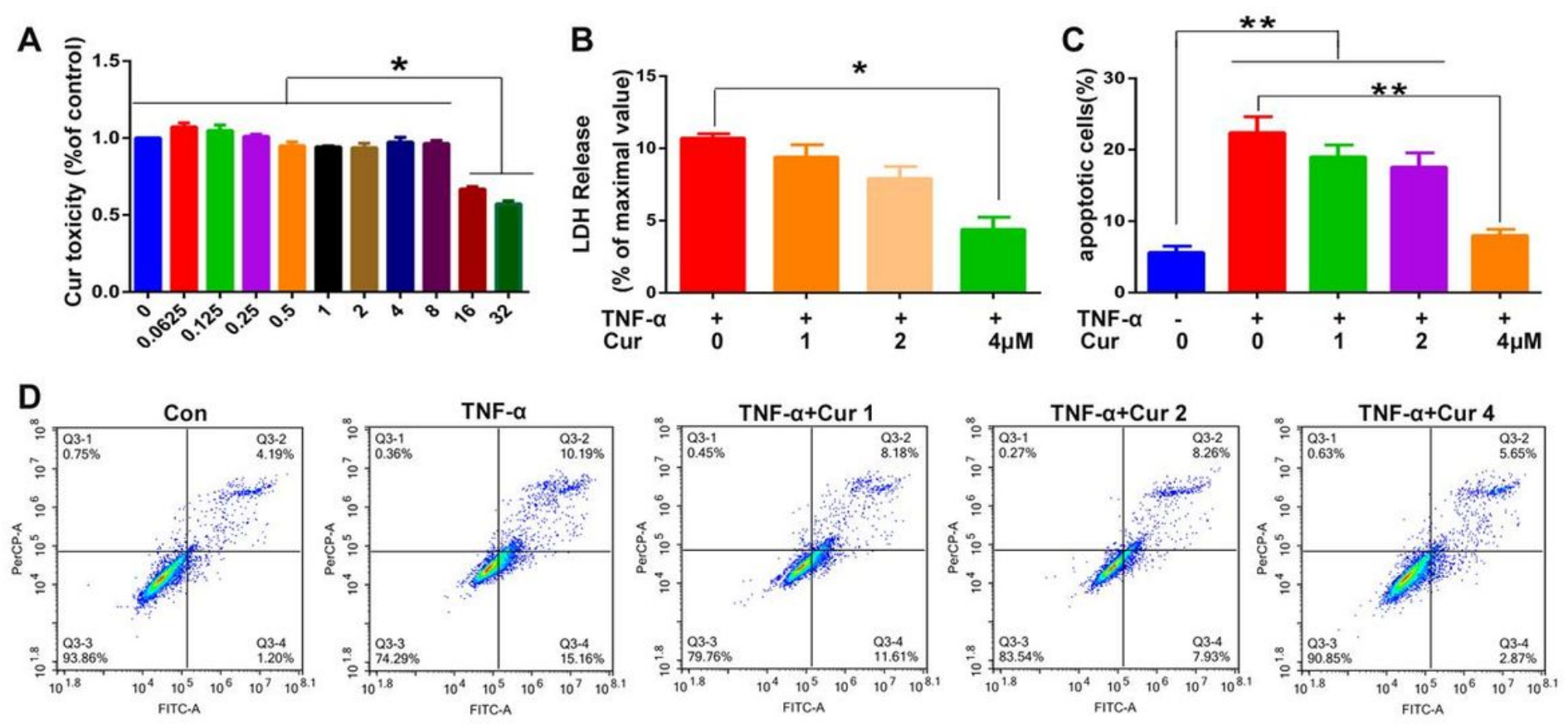

\section{Figure 2}

Curcumin inhibited apoptosis of hUC-MSC mediated by TNF- $a$. A. The CCK-8 assay was used to detect the drug toxicity within $24 \mathrm{~h}$ after different concentration of cur interference. B. The LDH release test for the anti-apoptotic of cur on hUC-MSC mediated by TNF- $\alpha$. The statistical analysis (C) and representative flow cytometry (D) for the anti-apoptotic effect of cur on hUC-MSC mediated by TNF- $a .{ }^{*} P<0.05$, ** $\mathrm{P}<0.01, \mathrm{~N}=5$ /group. 


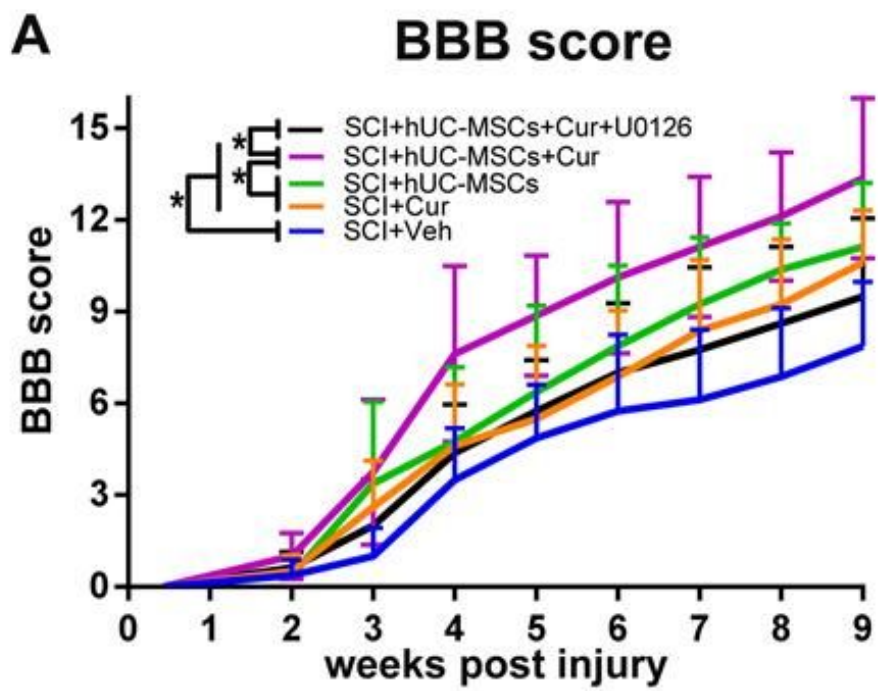

C

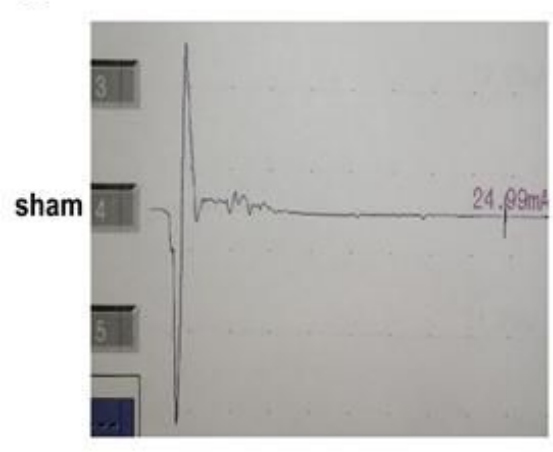

B inclined plane test

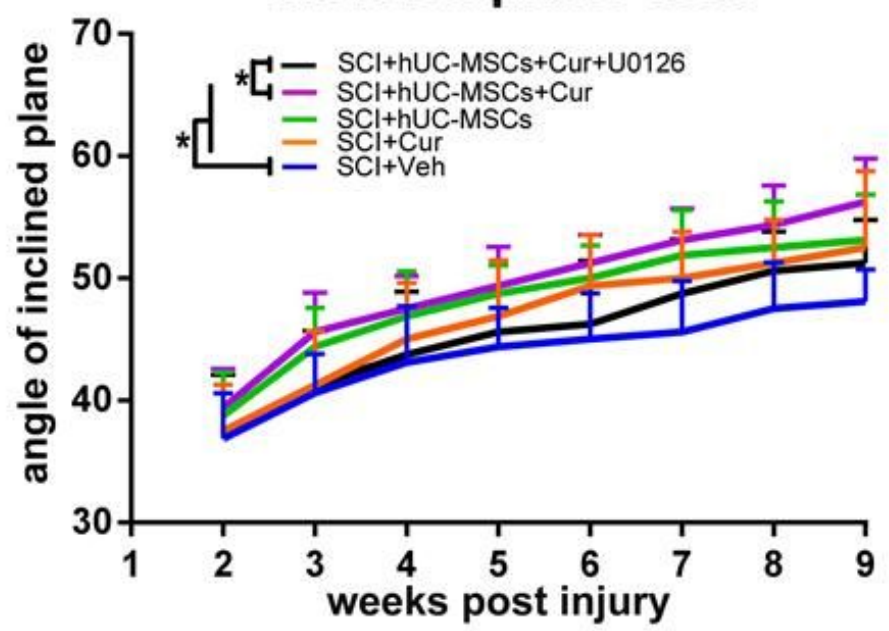

D

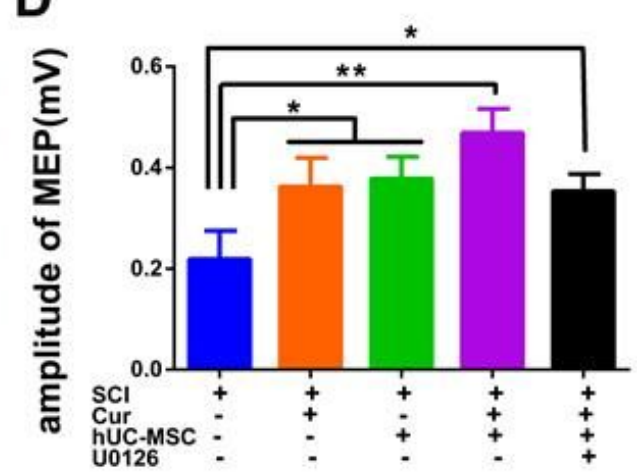

Figure 3

The expression of MAPK signal pathway in hUC-MSC with different concentration of cur interference. A. WB analysis for p42/44, JNK and p38. B-D. Statistical analysis of WB for $p 42 / 44$, JNK and $p 38$. * $P<0.05$, $N=5 /$ group. 
A
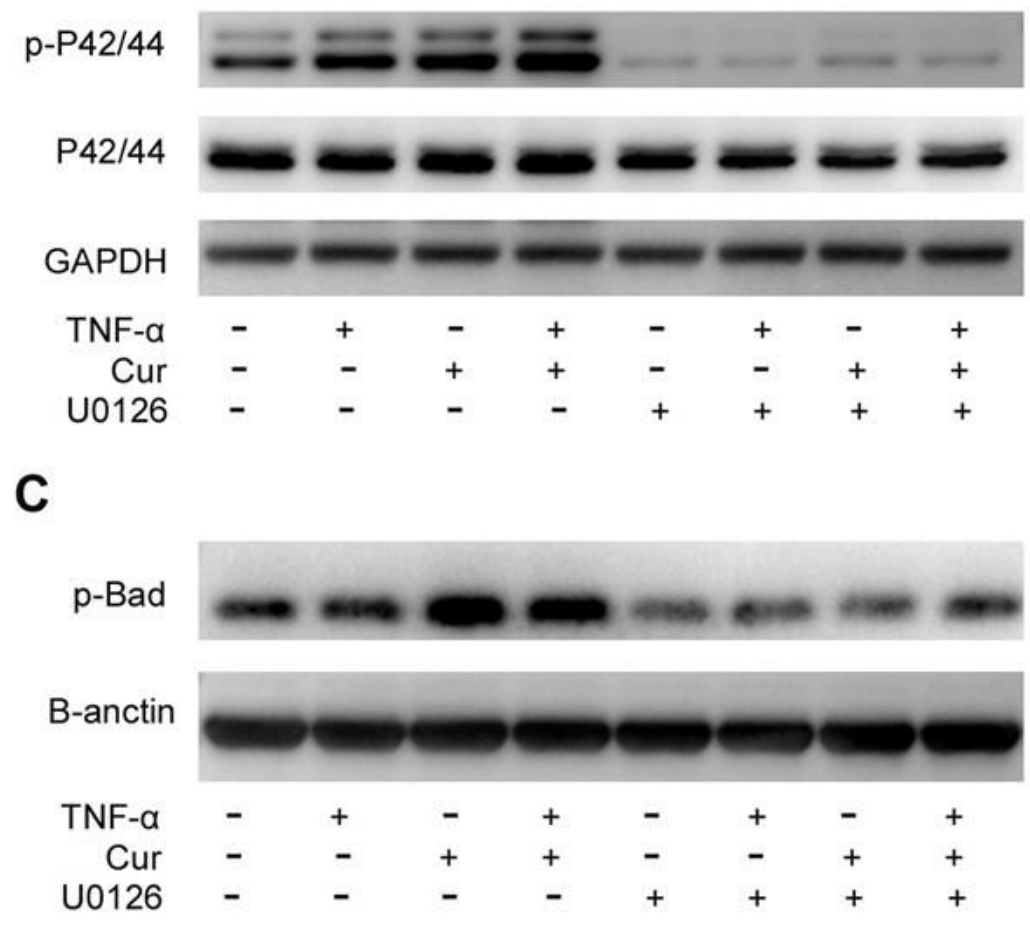
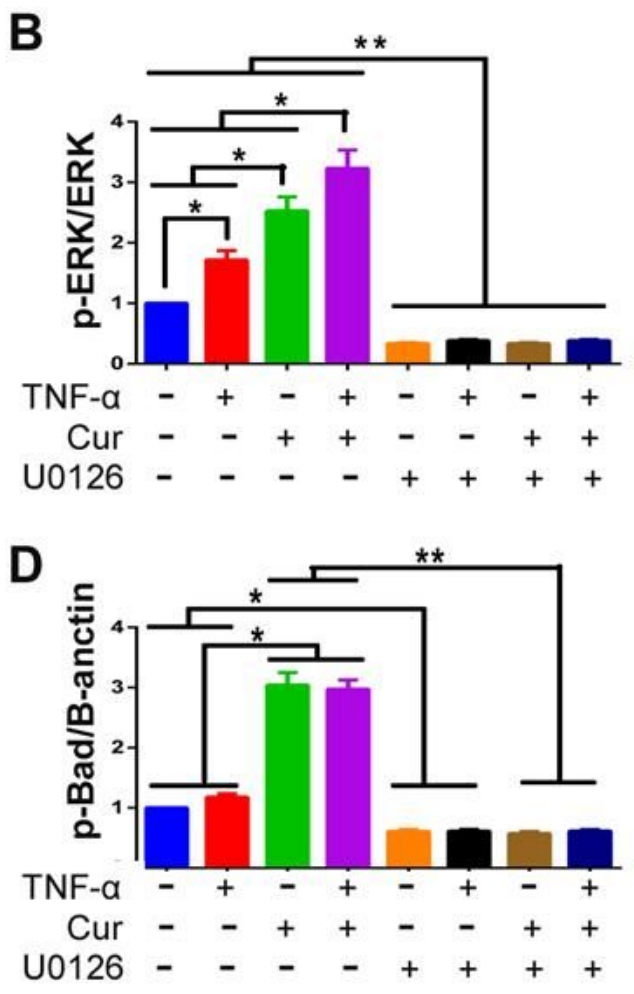

\section{Figure 4}

Curcumin suppressed hUC-MSC apoptosis through ERK/MAPK signal pathway. $5 \mathrm{ng} / \mathrm{ml}$ TNF-a was used to induce apoptosis of hUC-MSCs with or without ERK antagonists U0126 ten minutes followed by $4 \mathrm{uM}$ cur intervention for $24 \mathrm{~h}$. The WB analysis (A) and statistical analysis (B) for phosphorylation p42/44 (ERK) protein expression. The WB analysis (C) and statistical analysis (d) for apoptosis-related protein $p$ Bad expression. * $P<0.05$, ** $P<0.01, N=5 /$ group. 

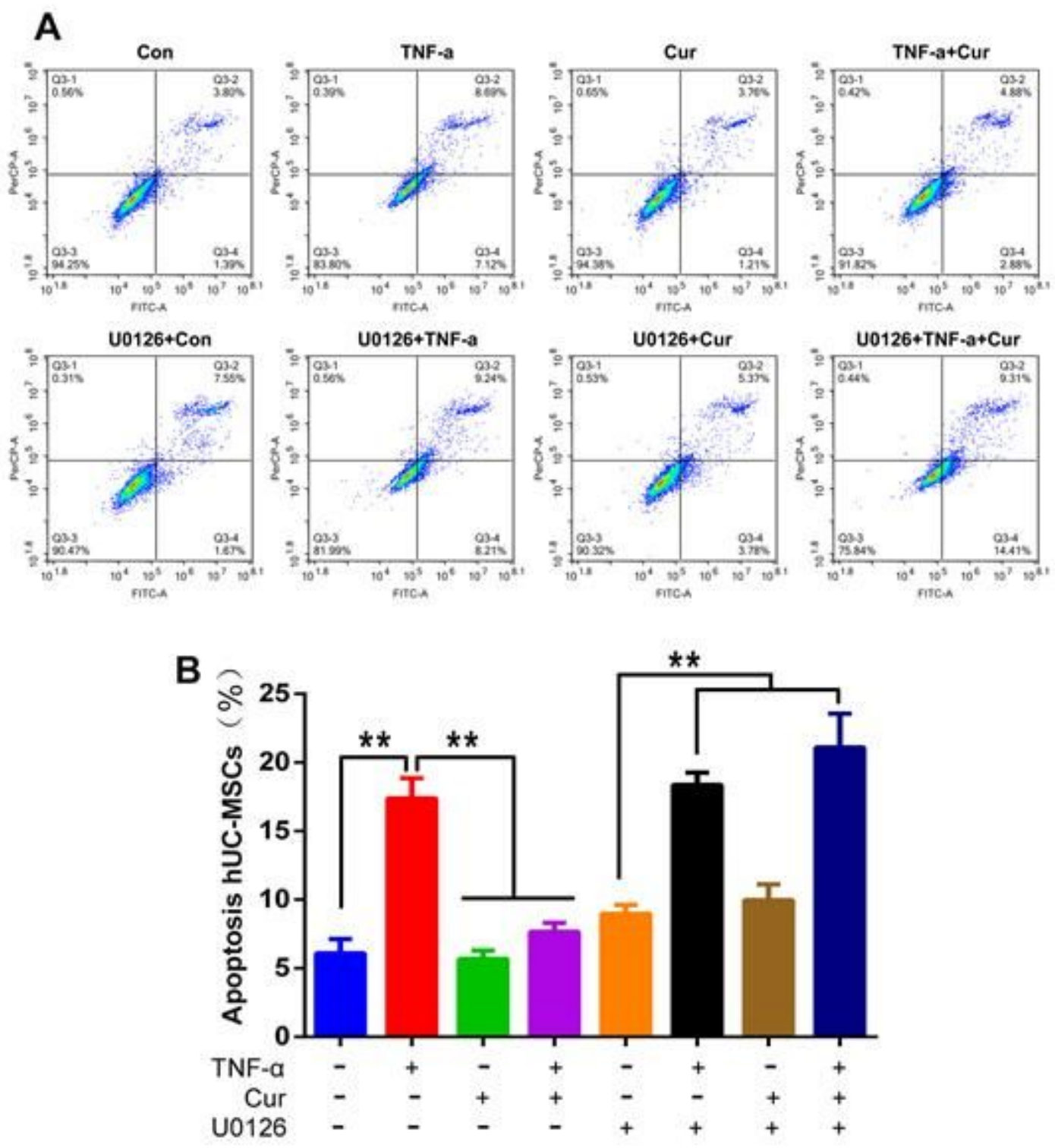

\section{Figure 5}

The flow cytometry for the anti-apoptotic effect of cur on hUC-MSC in vitro. $5 \mathrm{ng} / \mathrm{ml}$ TNF-a was used to induce apoptosis of hUC-MSCs with or without ERK antagonists U0126 ten minutes followed by 4uM cur intervention for $24 \mathrm{~h}$. The representative flow cytometry (A) and statistical analysis (B) for the antiapoptotic effect of cur on hUC-MSC mediated by TNF- $a$. ** $P<0.01, N=5$ /group. 
A
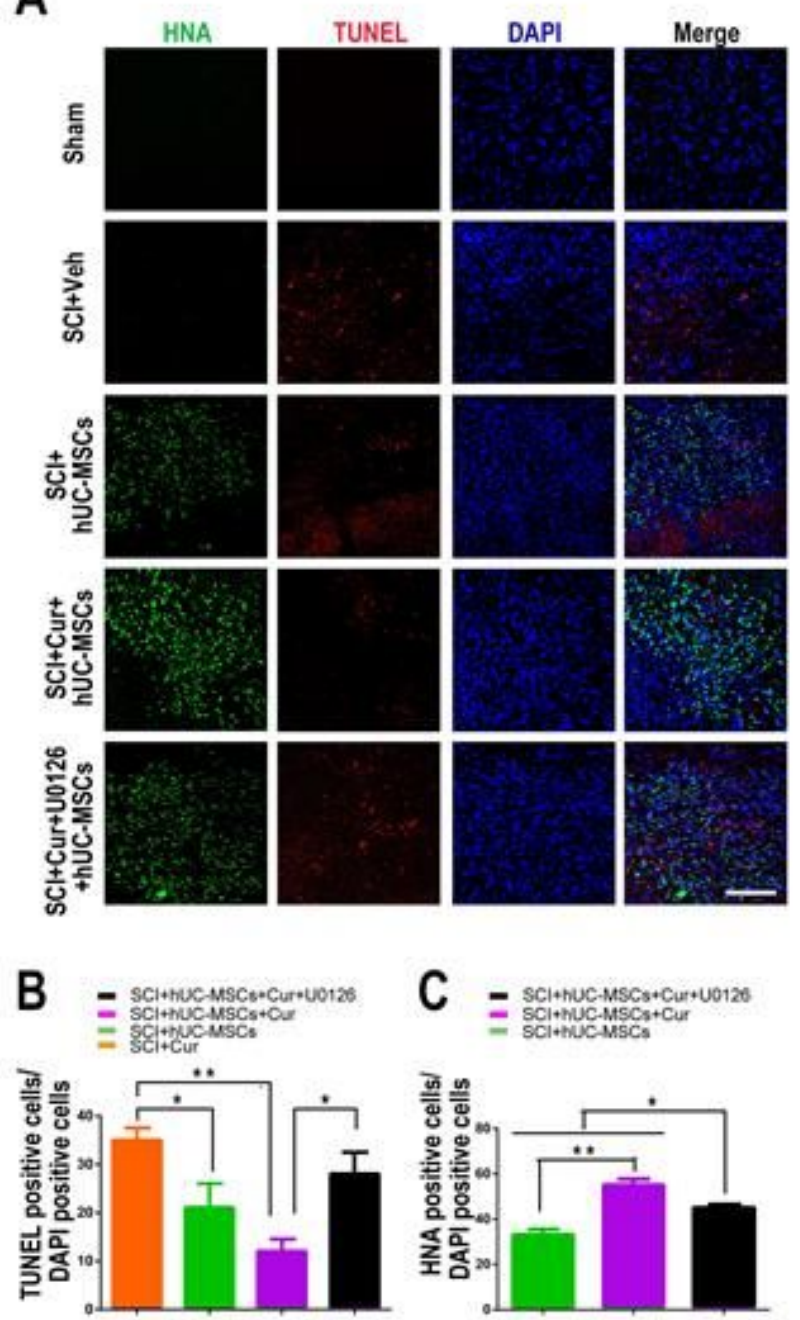
C $\equiv$

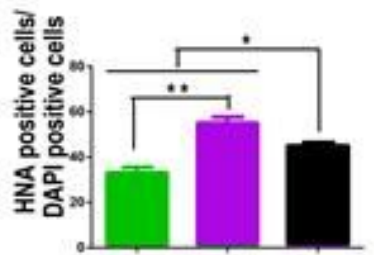

D
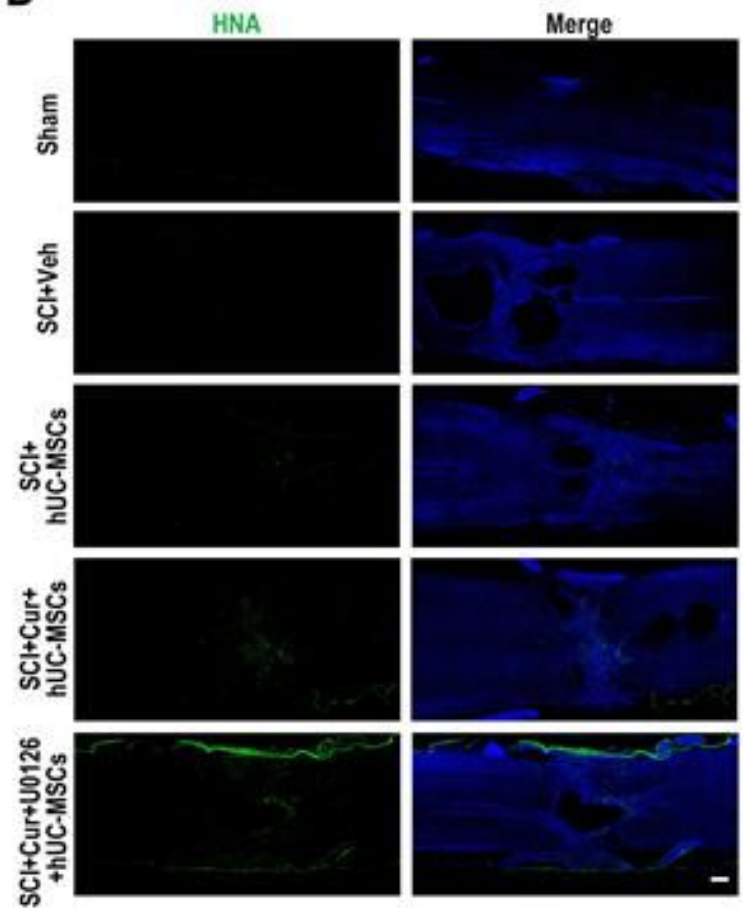

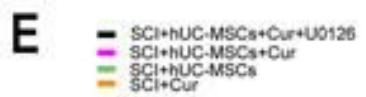
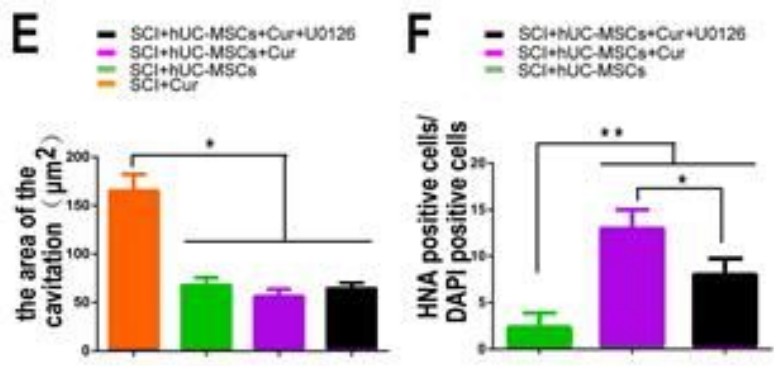

\section{Figure 6}

Curcumin reduced the transplanted hUC-MSC apoptosis in the injured region through ERK/MAPK signal pathway. A. The representative TUNEL staining for hUC-MSCs apoptosis in the injured region 3 days post hUC-MSC transplantation after SCI. (B-C) The statistical analysis for apoptotic hUC-MSCs cell and viable hUC-MSCs cell with or without U0126 combined cur 3 days post transplantation. D. The representative immunofluorescence analysis for hUC-MSCs apoptosis in the injured region 8 weeks post hUC-MSC transplantation with TUNEL staining. (E-F) The statistical analysis for apoptotic hUC-MSCs cell and viable hUC-MSCs cell with or without U0126 combined cur 8 weeks post transplantation. bar $=20 \mu \mathrm{m}$, * $P<0.05$, ** $P<0.01, N=5 /$ group. 


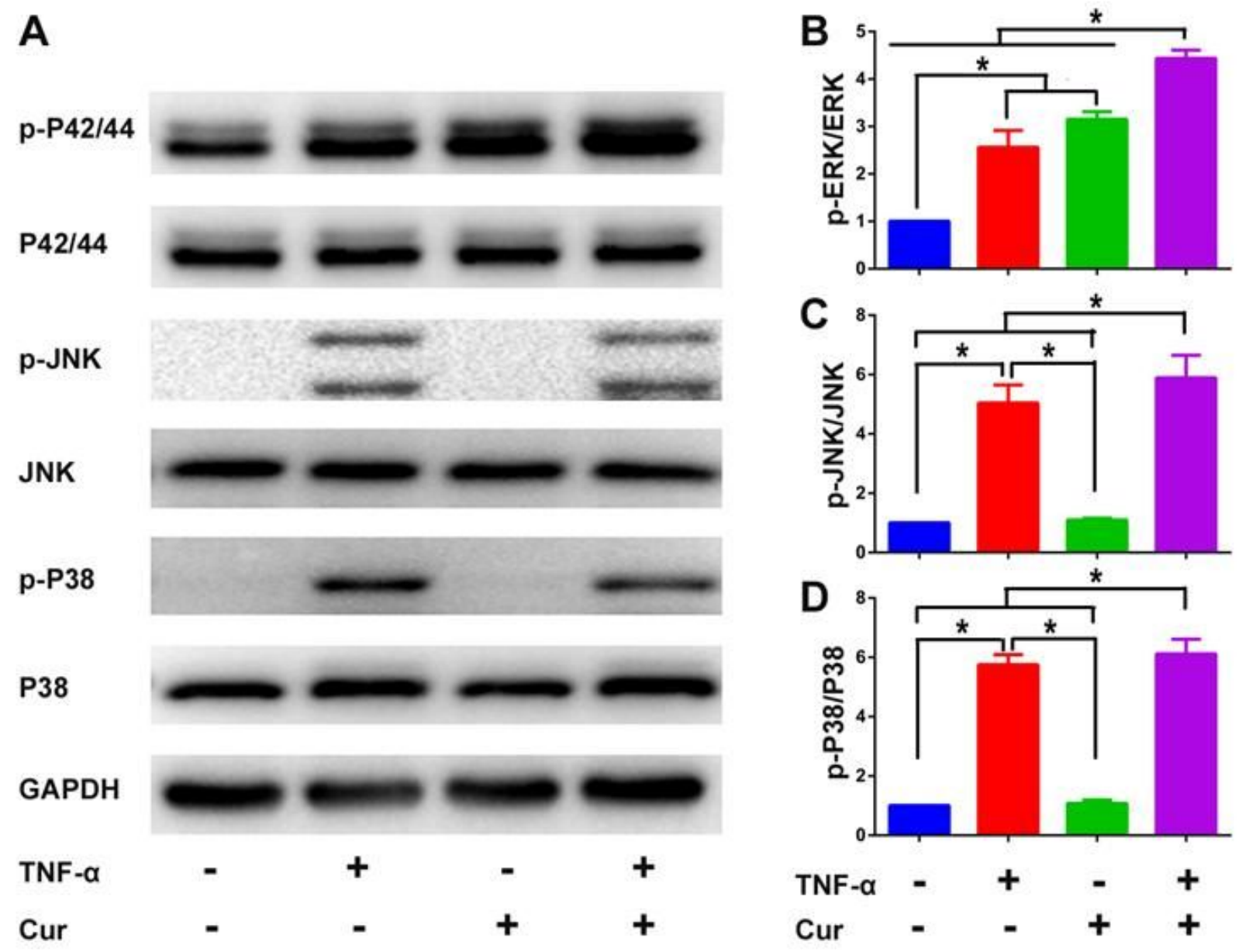

Figure 7

Curcumin combined with hUC-MSC promoted the recovery of hindlimb function in rats after SCl. A. the assessment of the motor function recovery with the BBB scale (A) and inclined plate test (B). Wave shapes(C) and statistical analysis (D) for motor-evoked potential (MEP) of each group at 8 weeks post hUC-MSC transplantation. * $\mathrm{P}<0.05$, ${ }^{* *} \mathrm{P}<0.01, \mathrm{~N}=5$ /group. 